\title{
Efficacy of Different Drugs in Treating Urinary Schistosomiasis: Systematic Review and Network Meta-analysis
}

\author{
Huilong Fang ${ }^{1}$, Xuli Guo ${ }^{1}$, Chuwei Tang ${ }^{2}$, Fuchun Chen1, Feng Luo ${ }^{1}$, Junjie Wang ${ }^{3^{*}}$ \\ ${ }^{1}$ Department of Pathogenic Biology and Immunology Xiangnan University, Chenzhou, China \\ ${ }^{2}$ Basic Medical College, Xiangnan University, Chenzhou, China \\ ${ }^{3}$ Department of Pharmacology Xiangnan University, Chenzhou, China
}

\begin{abstract}
This review compares the efficacy of three drugs, namely praziquantel (PZQ), artesunate, and metrifonate in urinary schistosomiasis. Databases were searched for articles comparing the effectiveness of any of the three drugs to other medications or controls in urinary schistosomiasis in children aged 18 or less. Stata software was opted to generate the network meta-analysis. Efficacy (Cure rate and egg reduction rate) was the main outcome measure. Pairwise and network meta-analysis were used to report Odds Ratios (ORs) with either 95\% confidence interval (CI) for direct comparisons or $95 \%$ credible intervals (CrI) for indirect comparisons. The SUCRA plot for cure rate revealed that PZQ (SUCRA=40.4\%) was the fourth effective drug after albendazole $400 \mathrm{mg}$ (SUCRA=71.5), metrifonate $5 \mathrm{mg}$ (SUCRA=62.2\%), and metrifonate $10 \mathrm{mg}$ (SUCRA= 59.7). PZQ was only superior to metrifonate $7.5 \mathrm{mg}$. ORs were PZQ $40 \mathrm{mg}$ (OR 0.48; 95\% CI -3.55 to 4.51; p-value 0.816), artesunate $6 \mathrm{mg}$ (OR 0.06; 95\% CI -5.67 to 5.79; p-value 0.983), metrifonate $5 \mathrm{mg}$ (OR -1.65; 95\% CI-7.52 to 4.21; p-value 0.581 ), metrifonate $10 \mathrm{mg}$ (OR -1.76; 95\% CI -8.86 to 5.34; p-value 0.628 ) and metrifonate $7.5 \mathrm{mg}$ (OR -2.40; $95 \% \mathrm{CI}$-9.78 to 4.98; p-value 0.524). A similar plot for egg reduction rate showed an exclusive superiority of PZQ $40 \mathrm{mg}$ (SUCRA $=94.4 \%$ ), followed by metrifonate 10mg (SUCRA $=82.3 \%$ ) and niridazole $25 \mathrm{mg}$ plus metrifonate $10 \mathrm{mg}$ (SUCRA $=48.6 \%$ ). Our network analysis revealed that PZQ $40 \mathrm{mg}$ was the most efficient drug in reducing egg count, whereas albendazole $400 \mathrm{mg}$ showed the highest cure rates.
\end{abstract}

KEYWORDS: Urinary schistosomiasis; Praziquantel; Artesunate; Metrifonate

\section{INTRODUCTION}

Urinary schistosomiasis is a chronic parasitic infection caused by trematodes of the genus Schistosoma known as Schistosoma haematobium (SH). SH, also known as "urinary blood fluke", inhabits and produces eggs in the small venules of the perivesical and portal systems. Urinary schistosomiasis is endemic in 53 countries in Africa and the Middle East, where more than 110 million people are infected $[1,2]$. Noteworthy, the prevalence reaches its peak among school-age children to be as high as $46.5 \%[3,4]$. A myriad of medications was evaluated for their efficacy in treating SH infection [5-15]. Praziquantel (PZQ) is the most used drug worldwide and is the drug of choice for controlling schistosomiasis in endemic regions [16]. The reasons for that is its efficacy in reducing egg count at a relatively high rate across different types of schistosomiasis [17]. Moreover, PZQ is has shown good safety profiles making it the drug of choice for children and pregnant women [18,19]. Additionally, PZQ has a low risk of noncompliance because it is administered as a single oral dose of $40 \mathrm{mg} / \mathrm{kg}$ body weight [20]. Noteworthy, recent literature questioned this treatment regimen and suggested that PZQ in multiple dosages is more effective than a single dose [21].

"Correspondence to: Junjie Wang, Department of Pathogenic Biology and Immunology Xiangnan University, Chenzhou, China, Tel: 18566267394; E-mail: 475911403@qq.com

Received: January 18, 2021; Accepted: Febuary 04, 2021; Published: Ferbuary 12, 2021

Citation: Fang H, Guo X, Tang C, Chen F, Luo F, Wang J. (2021) Efficacy of Different Drugs in Treating Urinary Schistosomiasis: Systematic Review and Network Meta-analysis. Trop Med Surg. 9:231. doi: 10.35248/2329-9088.8.2.231

Copyright: $\odot 2021$ Fang H, et al. This is an open-access article distributed under the terms of the Creative Commons Attribution License, which permits unrestricted use, distribution, and reproduction in any medium, provided the original author and source are credited. 
In the same context, artesunate, originally an antimalarial treatment, has proved efficacy and tolerability as an antischistosomal therapy [5,22]. Two doses of artesunate were more cost-effective compared to single-dose PZQ [23]. Nonetheless, PZQ-artesunate combination was safer and more effective as opposed to using either drug [24,25]. Metrifonate, a cholinesterase inhibitor, is another selective treatment for $\mathrm{SH}$; it has good efficacy and safety profiles [26]. Although it is economic and causes low recurrence rates, the complicated dose regimen may limit its use [27].The reason for such complexity is the time needed for cholinesterase to return to its normal level, which may take 8-15 days [28]. Moreover, three doses of metrifonate are needed to produce the same effect as one dose of PZQ in terms of egg reduction rates [29]. Another limitation in using metrifonate is that a prophylactic dose is needed within two years to guard against recurrence [30]. Niridazole is also highly effective for treating schistosomiasis, and a single dose of niridazole $25 \mathrm{mg} / \mathrm{kg}$ daily has a high cure rate [11,31]. However, it has serious side effects in susceptible individuals including central nervous system toxicities and allergic reactions, which limit its use compared to other safe alternatives [32].

In this study, we aim to compare efficacy (cure rate and egg reduction rate) of three drugs, namely praziquantel, artesunate, and metrifonate in cases of $\mathrm{SH}$.

\section{METHODOLOGY}

\section{Search strategy and study selection}

The study was conducted following the accepted methodology recommendations of the PRISMA checklist for systematic reviews. On December 18, 2018, we searched PubMed, Cochrane Central, Scopus, Web of Science, and Ovid databases for pertinent English articles using search terms ("Schistosoma haematobium" or "schistosomiasis haematobia" or "Bilharzia haematobium" or "urinary schistosomiasis" or "urogenital schistosomiasis" or "vesical schistosomiasis" or "Bilharziasis haematobium" or "Bilharzia haematobium") and (Metrifonate or "Praziquantel" or "Artesunate" or anthelmin* or treat* or therapy*). A manual search was done by searching for relevant publications in references of included articles; relevant papers in PubMed and Google Scholar; and primary studies that had cited the included papers. We also hand-searched using each keyword to avoid missing any relevant publications.

\section{Study selection}

Three independent reviewers scanned the titles and abstracts to select potentially relevant articles. We included all original studies reporting treatment of urinary schistosomiasis. There was no restriction on country, language, or publication date. We excluded papers if they met the following exclusion criteria:

- in vitro or animal studies,

- data duplication, overlapping or unreliably extracted or incomplete data,

- unoriginal work including abstract only articles, reviews, theses, books, conference papers or articles with in available full texts (editorials, author responses, letters, and comments), and

- any previous systematic reviews, meta-analyses, and literature reviews on our topic of interest.

Three reviewers independently performed an initial eligibility assessment of the retrieved titles and abstracts. Full texts of eligible articles were then retrieved and reviewed for inclusion. In both screening steps, inclusion, or exclusion of a study by all three reviewers was considered conclusive. Conflicts were resolved through discussion among the authors. When necessary, the authors sought the opinion of senior reviewers on disagreements and discrepancies.

\section{Data extraction}

Based on a pilot scan and extraction, two authors prepared a data extraction sheet using Microsoft Excel, and three reviewers independently extracted data from included studies. For accuracy, two different authors revised the data, and a third reviewer rechecked them. Disagreements or discrepancies were resolved through discussion and reaching consensus. Papers by the same research group and those studying the same factors were checked for potential duplicate data based on recruitment year, recruitment place, and confirmation from study authors. The outcome of interest was the efficacy of the medications of concern (PZQ, artesunate, and metrifonate), and outcome measurements were opted premised on the most commonly reported data in the included papers, namely cure rates, and egg reduction rates. Not only were these two measures endorsed for being frequently reported but also because they were feasible to assess pre- and post-therapy using diagnostic tests. Two independent reviewers extracted and recapitulated data entailing study ID, last name of the first author, publication year, country, total number of participants, percentage of males, age touted as mean (SD), and the administered medications (name, dose, number of participants assigned to each drug). Regarding tools of outcome measurement, the extracted data encompassed drug name, the quantitative mean of efficacy, the total number of patients, the dose, the length of the therapeutic course in days, and the diagnostic test used for assessment. Whenever any article reported multiple checkpoints, only the last point was analyzed.

\section{Quality assessment}

Two reviewers have independently assessed the risk of bias of the included studies using the National Institute of Health (NIH) Quality Assessment Tool for Observational Cohort and Cross-sectional Studies and Case-control Studies. The elements of the quality assessment were indexed either Yes (1), No (0), or others including CD (Cannot Determine), NA (Not Applicable), and NR (Not Reported). Eventually, papers were rated fair, good, or high based on the final score. Having a nonrandomized controlled trial included, the Risk of Bias in NonRandomized Studies of Interventions Tool (ROBINS-I) tool was used for its assessment [33]. This tool includes six categories: confounding bias, participants selection bias, the bias in classification of interventions, bias due to deviations from intended interventions, bias due to missing data, and bias in the 
measurement of outcomes. The questions under each category were assigned either Y (Yes), N (No), PY (Probably Yes), or NI (No Information), and so was the case for the overall bias.

\section{Statistical analysis}

Odds Ratios (ORs) with 95\% confidence interval (CI) for direct comparisons or $95 \%$ credible intervals $(\mathrm{CrI})$ for indirect comparisons were used. A network meta-analysis was performed using Stata software (version 14.2, StataCorp, College Station, TX) with random-effects models. To rank the treatments, the surface under the cumulative ranking probabilities (SUCRA) were opted to show which treatment was the best. Heterogeneity was considered significant with either $\mathrm{I} 2>50$ or $\mathrm{P}<0.05$ with a subsequent adjustment of the model to small-study effects (incorporation of the heterogeneity) in this case. Inconsistency was also considered significant with either $\mathrm{P}<0.05$, which was further investigated using node splitting methods (of direct and indirect comparisons) whenever indicated.

\section{RESULTS}

\section{Search results and screening process}

Our search retrieved 2303 studies with only 1434 left after removing duplicates. Title and abstract screening using the criteria left only 89 studies eligible for further full-text screening. Following the full-text screening, 20 studies were included in both quantitative and qualitative synthesis. The manual search retrieved no additional relevant studies. Figure 1 shows a summary of the search and screening process.

\section{Characteristics and quality of included studies}

The patient characteristics and the designs of the included studies. Apart from two studies of poor quality, most of the studies were either good or fair in quality.

\section{Cure rate}

For cure rates, six different treatments/doses were compared, and the network plot is showcased. Pooling direct and indirect comparisons showed an advantage of albendazole $400 \mathrm{mg}$ (SUCRA=71.5) over praziquantel (SUCRA=40.4); different doses of metrifonate (SUCRA $=62.2,59.7$, and 38 for $5 \mathrm{mg}, 10$ $\mathrm{mg}$ and $7.5 \mathrm{mg}$ respectively); and artesunate (SUCRA=28.2). There was significant heterogeneity $(2=2.78, \mathrm{I} 2=71.55 \%)$ with no significant inconsistency $(\mathrm{P}=0.06)$. Moreover, adjusting our model to small-study effects (incorporation of the heterogeneity) did not materially alter the relative effectiveness and the ranking of treatments. In the same context, pairwise comparisons of all drugs to albendazole $400 \mathrm{mg}$ revealed that albendazole was more effective than any other treatment/dose. However, this difference in cure rates was not statistically significant throughout all comparisons.

\section{Egg reduction rate}

A network plot of the comparisons of egg reduction among eight different treatments/doses. Pooling direct and indirect comparisons showed an obvious advantage of PZQ $40 \mathrm{mg}$
(SUCRA=94.4), followed by metrifonate $10 \mathrm{mg}($ SUCRA=82.3) and then niridazole $25 \mathrm{mg}$ plus metrifonate $10 \mathrm{mg}$ (SUCRA =48.6) (Detailed SUCRA scores). There was a significant heterogeneity $(2=1.21, \mathrm{I} 2=65.73 \%)$ and significant inconsistency $(\mathrm{P}=0.007)$. Moreover, adjusting our model to small-study effects (incorporation of the heterogeneity) did not materially alter the relative effectiveness and ranking of treatments. In the same context, pairwise comparisons of all drugs to Placebo revealed Praziquantel $40 \mathrm{mg}$-Artesunate $4 \mathrm{mg}$ as a combination was the best, followed by Praziquantel $40 \mathrm{mg}$ and the Niridazole $25 \mathrm{mg}$-Metrifonate $10 \mathrm{mg}$ combination. Interestingly, this difference in cure rate was statistically significant across all drugs when compared to placebo.

\section{DISCUSSION}

This study is, to the best of our knowledge, the first network meta-analysis of its kind to assess the efficacy of all proposed treatments for urinary schistosomiasis in the literature through direct and indirect comparisons between the various treatment modalities. Our network analysis revealed that albendazole (400 $\mathrm{mg}$ ) achieved higher percentages of cure rate than other treatment modalities. Albendazole is used commonly for treating some human worm infections. Recently, it has been combined with praziquantel for the management of schistosomiasis and other soil-transmitted helminthiases [34,35].

Noteworthy, albendazole has never been investigated as monotherapy for $\mathrm{SH}$, but one study reported the outcomes of a single dose of combined praziquantel and albendazole and concluded that albendazole impacts the effect of praziquantel [36]. Olds et al. have reported a failure rate (defined as the continued detection of parasite eggs 45 days after treatment of $\mathrm{SH}$ (of $79.6 \%$ in patients treated with Albendazole alone. This percentage nosedived to $38.5 \%$ after adding praziquantel to the therapy [36]. Furthermore, they reported a failure rate of $35.1 \%$ in praziquantel alone, which is still much better than albendazole alone. Olds et al. had a few limitations: First, they excluded female patients who were liable to pregnancy because albendazole is a known teratogenic. Accordingly, most of their patients were on praziquantel, which might have made their analysis standing in favour of praziquantel. Second, they did not provide any data on the characteristics of their study participants, the intensity of the infection, or the number of patients on each treatment modality. Another trial showed higher cure rates in praziquantel than cure rates of albendazole alone $(78 \%$ vs $66 \%)$ but with statistically insignificant difference. Additionally, the mean post-treatment egg count was lower in patients on praziquantel compared to those on albendazole $(2.0 \pm 1.2$ and $2.7 \pm 1.3$, respectively) [37]. Even though the reports indicate that praziquantel is more efficient than albendazole in treating urinary schistosomiasis, albendazole is more readily available and cost-effective [37].

Our finding regarding the efficacy of PZQ is inconsistent with the recommendations in the literature. The World Health Organization recommends that schistosomiasis should be treated with single-dose PZQ of at least $40 \mathrm{mg} / \mathrm{kg}$ [20]. Similarly, the recently updated review by Kramer et al. [24] supports the current evidence of applying $40 \mathrm{mg} / \mathrm{kg}$ praziquantel to patients 
with urinary schistosomiasis. One major limitation to the study by Kramer et al., besides being mostly qualitative review with only minimal meta-analyses, is that all their included trials were conducted in Sub-Saharan Africa, except for one conducted in Saudi Arabia. So, their conclusions cannot be generalized. On the other hand, our anthem lysis revealed that PZQ held fourth record for cure rates after albendazole, metrifonate $(5 \mathrm{mg} / \mathrm{kg})$, and metrifonate $(10 \mathrm{mg} / \mathrm{kg})$. Herein, we propose multiple explanations for this finding. The low cure rates of PZQ could be a possibly increasing PZQ resistance in the assessed trials as observed by some authors [38-42]. Praziquantel is also kills the adult worms, not the immature stages; therefore, most of our patients on PZQ might have been harbouring the immature stages at treatment time.

In our analysis, even though praziquantel was ranked fourth in cure rates, it was ranked first in egg reduction rates. There were no data available on egg count reduction in patients on albendazole; thus, it was not included in the analysis. Future studies should assess thoroughly the changes in egg count in patients on albendazole. On the other hand, a combination of niridazole and metrifonate was ranked second in our analysis, but one should keep in mind that both drugs are old, unavailable, and are no longer used in practice. Of note, egg count reduction is not an overly sensitive parameter of clinical and parasitological cure since negative urine examinations is not an indicator of cure. The literature reported that the absence of eggs in the urine is only an occasional finding and does not necessarily exclude infection [43]; accordingly, repeated urine examination after treatment is more useful in diagnosing persistent excretion of eggs in the urine and controlling any the possible transmission of the infection. Cystoscopy, in addition to biopsy and histopathological evaluation, is a pivotal indicator of parasitological cure [43]. Therefore, extra caution should be given to the interpretation of the data on egg count as they do not necessarily reflect the efficacy of treatments.

\section{STRENGTHS AND LIMITATIONS}

The main strength of our study is being, to the best of our knowledge, the first network meta-analysis to assess the direct and indirect comparisons among different treatment modalities for urinary schistosomiasis in terms of cure and egg reduction rates. Also, unlike the recent Cochrane review of Kramer et al. [24], we assessed the efficacy of albendazole in treating urinary schistosomiasis. However, we encountered several limitations, the biggest of which is the lack of data regarding the egg count reduction in patients on albendazole, and that restricted us from including the egg count networking. Also, the definition of cure differed among the included trials, which impeded us from reaching a sheer conclusion on the best treatment for urinary schistosomiasis. Moreover, the intensity of infection was not reported in most trials; the thing that might have affected the results of egg count reduction to somewhat. Therefore, these issues should be addressed in future work.

\section{CONCLUSION}

Our network analysis revealed that albendazole is the most efficient drug with the highest cure rates in cases of urinary schistosomiasis, whereas PZQ took fourth place. As regards the percentage of egg count reduction, PZQwas superior to other medications.

\section{CONFLICT OF INTEREST}

The authors declare that they have no conflict of interest.

\section{FUNDING}

None

\section{REFERENCES}

1. Barakat RM. Epidemiology of Schistosomiasis in Egypt: Travel through Time: Review. J Advanced Res. 2013;4:425-432.

2. Olveda DU, Li Y, Olveda RM, Lam AK, Chau TN. Bilharzia: Pathology, Diagnosis, Management and Control. Trop Med Sur. $2013 ; 1$.

3. Degarege A, Mekonnen Z, Levecke B, Legesse M, Negash Y. Prevalence of Schistosoma haematobium Infection among SchoolAge Children in Afar Area, Northeastern Ethiopia. PLOS ONE. 2015;10:e0133142-e0133142.

4. Ismail HAHA, Hong S-T, Babiker ATEB, Hassan RMAE, Sulaiman MAZ. Prevalence, risk factors, and clinical manifestations of schistosomiasis among school children in the White Nile River basin, Sudan. Parasites vectors. 2014;7:478-478.

5. Borrmann S, Szlezak N, Faucher JF, Matsiegui PB, Neubauer R. Artesunate and praziquantel for the treatment of Schistosoma haematobium infections: a double-blind, randomized, placebocontrolled study. J Inf Dis. 2001;184:1363-1366.

6. Coulibaly JT, Panic G, Yapi RB, Kovac J, Barda B. Efficacy and safety of ascending doses of praziquantel against Schistosoma haematobium infection in preschool-aged and school-aged children: a single-blind randomised controlled trial. BMC Med. 2018; $16: 81$.

7. Feldmeier H, Chitsulo L. Therapeutic and operational profiles of metrifonate and praziquantel in Schistosoma haematobium infection. Arzneimittelforschung. 1999;49:557-565.

8. Feldmeier H, Doehring E, Daffala AA, Omer AH, Dietrich M. Efficacy of metrifonate in urinary schistosomiasis: comparison of reduction of Schistosoma haematobium and S. mansoni eggs. The American Journal of Trop Med Hyg. 1982;31:1188-1194.

9. Kramer CV, Zhang F, Sinclair D, Olliaro PL. Drugs for treating urinary schistosomiasis. Cochrane Database of Syst Rev. 2014.

10. Moczon T, Swiderski Z. Schistosoma haematobium: histochemistry of glycogen, glycogen phosphorylase and glycogen branching enzyme in niridazole-treated females. Int J Parasitology. 1992;22:55-63.

11. Rey JL, Sellin E, Sellin B, Simonkovich E, Mouchet F. Comparative efficacy of oltipraz (1 dose, $30 \mathrm{mg} / \mathrm{kg})$ and the combination of niridazole $(25 \mathrm{mg} / \mathrm{kg})$ and metrifonate $(10 \mathrm{mg} / \mathrm{kg})$ against S. haematobium. Med Trop. 1984;44:155-158.

12. Shehata MA, Chama MF, Funjika E. Prevalence and intensity of Schistosoma haematobium infection among schoolchildren in central Zambia before and after mass treatment with a single dose of praziquantel. Trop Parasitology. 2018;8:12-17.

13. Wang XY, He J, Juma S, Kabole F, Guo JG. Efficacy of Chinamade praziquantel for treatment of Schistosomiasis haematobium in Africa: A randomized controlled trial. PLoS Negl Trop Dis. 2019;13:e0007238. 
14. Wilkins HA, Moore PJ. Comparative trials of regimes for the treatment of urinary schistosomiasis in The Gambia. J Trop Med Hyg. 1987;90:83-92.

15. Woldegerima E, Bayih AG, Tegegne Y, Aemero M, Jejaw Zeleke A. Prevalence and Reinfection Rates of Schistosoma mansoni and Praziquantel Efficacy against the Parasite among Primary School Children in Sanja Town, Northwest Ethiopia. J Parasitology Res. 2019;3697216.

16. Dayan AD. Albendazole, mebendazole and praziquantel. Review of Non-clinical Toxicity and Pharmacokinetics. Acta Trop. 2003;86:141-159.

17. Zwang J, Olliaro PL. Clinical Efficacy and Tolerability of Praziquantel for Intestinal and Urinary Schistosomiasis - A Metaanalysis of Comparative and Non-comparative Clinical Trials. PLoS Neg Trop Dis. 2014;8:e3286-e3286.

18. Olds GR. Administration of Praziquantel to pregnant and lactating women. Acta Trop. 2003;86:185-195.

19. Fulford AJC, Webster M, Ouma JH, Kimani G, Dunne DW. Puberty and age-related changes in susceptibility to schistosome infection. Parasitology Today 14;1998.

20. Organization. Preventive chemotherapy in human helminthiasis: coordinated use of anthelminthic drugs in control interventions : a manual for health professionals and programme managers. World Health Organization Press, Geneva. 2006.

21. Shehata MA, Chama MF, Funjika E. Prevalence, and intensity of Schistosoma haematobium infection among schoolchildren in central Zambia before and after mass treatment with a single dose of praziquantel. Trop parasitology. 2018;8:12-17.

22. Li Q, Weina P. Artesunate: The Best Drug in the Treatment of Severe and Complicated Malaria. Pharmaceuticals (Basel, Switzerland). 2010;3:2322-2332.

23. Inyang-Etoh PC, Ejezie GC, Useh MF, Inyang-Etoh EC. Efficacy of artesunate in the treatment of urinary schistosomiasis, in an endemic community in Nigeria. Annals Trop Med Parasitology. 2004;98:491-499.

24. Kramer Cv ZFSD, Olliaro PL. Drugs for treating urinary schistosomiasis. Cochrane Database of Syst Rev. 2014.

25. Inyang-Etoh PC, Ejezie GC, Useh MF, Inyang-Etoh EC. Efficacy of a combination of praziquantel and artesunate in the treatment of urinary schistosomiasis in Nigeria. Transactions of The Royal Society of Trop Med Hyg. 2009;103:38-44.

26. Nordgren I, Bergstrfm M, Holmstedt B, Sandoz M. Transformation and Action of Metrifonate. 1978;41:31-41.

27. Davis A, Bailey DR. Metrifonate in Urinary Schistosomiasis. Bulletin of the World Health Organization. 1969;21:209-224.

28. Davis RP, Bailey DR. Effect of metrifonate on blood cholinesterases in children during the treatment of schistosomiasis. Bulletin of the World Health Organization. 1972;46:747-759.

29. Schutte CHJ, Visser PS, Pienaar R. The effectiveness of metrifonate (Bilarcil) in the treatment of a South African strain of Schistosoma haematobium. South Afr J Epid Inf. 1995;10:12-12.
30. Doehring E, Feldmeier H, Dafalla AA, Ehrich JHH, Vester U. Intermittent chemotherapy with trichlorfon (metrifonate) reverses proteinuria, hematuria, and leukocyturia in urinary schistosomiasis: results of a three-year field study. J Infs Dis. 1984;149:615-620.

31. Wolfe HL. Treatment of urinary schistosomiasis with niridazole (Ambilhar) in 576 African schoolchildren. The Lan. 1967;289:350-354.

32. Catto BA, Valencia CI, Hafez K, Fairchild EH, Webster LT. 4-Keto niridazole: a major niridazole metabolite with central nervous system toxicity different than niridazole. J Pharm Exp Therap. 1984;228:662-668

33. Sterne JA, Hernán MA, Reeves BC, Savović J, Berkman ND. ROBINS-I: a tool for assessing risk of bias in non-randomised studies of interventions. BMJ. 2016;355:14919-i4919.

34. Friis H, Mwaniki D, Omondi B, Muniu E, Thiong'o F. Effects on haemoglobin of multi-micronutrient supplementation and multihelminth chemotherapy: a randomized, controlled trial in Kenyan school children. Eur J Clin Nutr. 2003;57:573-579.

35. Zhang Y, Koukounari A, Kabatereine N, Fleming F, Kazibwe F. Parasitological impact of 2-year preventive chemotherapy on schistosomiasis and soil-transmitted helminthiasis in Uganda. BMC Med. 2007;5:27-27.

36. Olds GR, King C, Hewlett J, Olveda R, Wu G. Double-blind placebo-controlled study of concurrent administration of albendazole and praziquantel in schoolchildren with schistosomiasis and geohelminths. J Infec Dis. 1999;179:996-1003.

37. Ben SA, Useh MF. A comparative study on the efficacy of praziquantel and albendazole in the treatment of urinary schistosomiasis in Adim, Cross River State, Nigeria. Int Heal. 2017;9:288-293.

38. Groning E, Bakathir H, Salem A, Albert L, Fernández R. Effectiveness and tolerance of praziquantel in schistosomiasis. Rev cubana de med trop. 1985;37:215-215.

39. Herwaldt BL, Tao L-f, van Pelt W, Tsang VCW, Bruce JI. Persistence of Schistosoma haematobium infection despite multiple courses of therapy with praziquantel. Clin Infec Dis. 1995;20:309-315.

40. Hatz CF, Vennervald BJ, Nkulila T, Vounatsou P, Kombe Y. Evolution of Schistosoma haematobium-related pathology over 24 months after treatment with praziquantel among school children in southeastern Tanzania. Amer J Tropl Med Hyg. 1998;59:775-781.

41. Ernould JC, Ba K, Sellin B. Increase of intestinal schistosomiasis after praziquantel treatment in a Schistosoma haematobium and Schistosomamansoni mixed focus. Acta trop. 1999;73:143-152.

42. Liang YS, Coles GC, Doenhoff MJ. Detection of praziquantel resistance in schistosomes. Trop Med Int Heal. 2000;5:72-72.

43. Silva IMd, Thiengo R, Conceição MJ, Rey L, Lenzi HL (2005) Therapeutic failure of praziquantel in the treatment of Schistosoma haematobium infection in Brazilians returning from Africa. Memórias do Instituto Oswaldo Cruz. 2005;100:445-449. 